\title{
Enhanced Gain in Slow-Light Photonic Crystal Waveguides with Embedded Quantum Dots
}

Ek, Sara; Hansen, Per Lunnemann; Semenova, Elizaveta; Yvind, Kresten; Mørk, Jesper

Published in:

2011 Quantum Electronics Conference \& Lasers and Electro-Optics (CLEO/IQEC/PACIFIC RIM)

Link to article, DOI:

10.1109/IQEC-CLEO.2011.6194085

Publication date:

2011

Link back to DTU Orbit

Citation (APA):

Ek, S., Hansen, P. L., Semenova, E., Yvind, K., \& Mørk, J. (2011). Enhanced Gain in Slow-Light Photonic Crystal Waveguides with Embedded Quantum Dots. In 2011 Quantum Electronics Conference \& Lasers and Electro-Optics (CLEO/IQEC/PACIFIC RIM) (pp. 1015-1017). IEEE. https://doi.org/10.1109/IQECCLEO.2011.6194085

\section{General rights}

Copyright and moral rights for the publications made accessible in the public portal are retained by the authors and/or other copyright owners and it is a condition of accessing publications that users recognise and abide by the legal requirements associated with these rights.

- Users may download and print one copy of any publication from the public portal for the purpose of private study or research.

- You may not further distribute the material or use it for any profit-making activity or commercial gain

- You may freely distribute the URL identifying the publication in the public portal 


\title{
Enhanced Gain in Slow-Light Photonic Crystal Waveguides with Embedded Quantum Dots
}

\author{
Sara Ek, Per Lunnemann, Elizaveta S. Semenova, Kresten Yvind and Jesper Mørk \\ DTU Fotonik - Department of Photonics Engineering \\ Denmark Technical University, building345, DK.2800 Kongens Lyngby, Denmark \\ Presenting author e-mail address: saek@fotonik.dtu.dk
}

\begin{abstract}
We experimentally demonstrate enhanced gain in the slow-light regime of quantum dot photonic crystal waveguide slabs. These are promising results for future compact devices for terabit/s communication, such as compact optical amplifiers and mode-locked lasers.
\end{abstract}

\section{Introduction}

Photonic crystal (PC) line defect waveguides possess a propagating mode within the bandgap of the PC. Through careful design the light dispersion may be tailored to achieve slow light at certain frequencies $[1,2]$. The light-matter interaction is enhanced as the group velocity of the mode is reduced, and it has been suggested to use active PC waveguides to achieve ultra-compact amplifiers [3]. Such a device is desired for compact photonic chips and interconnects, e.g. for chip-to-chip or board-to-board links. To our knowledge there are no experimental demonstrations of gain in PC amplifiers. Here we observe, for the first time, a strong gain enhancement with the decrease of the group velocity in a PC semiconductor optical amplifier. The experimental study includes PC samples with quantum dots (QD) or quantum wells (QW) incorporated in the InGaAsP waveguide slab.

Both quantum dots and wells have become important gain materials as they are more easily inverted than bulk material and the emission wavelength can be precisely tuned by altering the composition and dimensions. The combination of $\mathrm{PC}$ and $\mathrm{QD} / \mathrm{QWs}$ allow for control over both the optical and electronic properties with many possibilities for future devices. There are several demonstrations of lasing in photonic crystal structures utilizing the effect of distributed feedback. The lasing threshold is reduced due to the longer photonic lifetime for the slow light. This has been achieved by realizing a high-Q cavity [4], introducing composite defects [5], or creating band edge lasing microcavities [6-8]. There are a few contributions in the literature showing lasing explained by gain enhancement at low group velocities $[9,10]$.

In this contribution there are no cavities in the $1 \mathrm{~mm}$ long PC waveguides, and the residual reflections at the facets are suppressed using inverted tapers. Transmission measurements on the optically pumped active PC waveguides show enhanced gain for the slow light wavelengths close to the photonic band edge. This holds great promise for the realization of efficient, ultra-compact PC optical amplifiers in the near future.

\section{Device fabrication and characteristics}

The fabricated device is based on the InP-material system, which is commonly used for communication applications operating in the $1.33 / 1.55 \mu \mathrm{m}$ regime. Three InGaAsP/InP epitaxial wafers were grown for this experiment; one single QW, one multi (10) QW, and one with three layers of QD. The central emission wavelengths for the three wafers were at 1525,1530 and $1565 \mathrm{~nm}$ respectively.

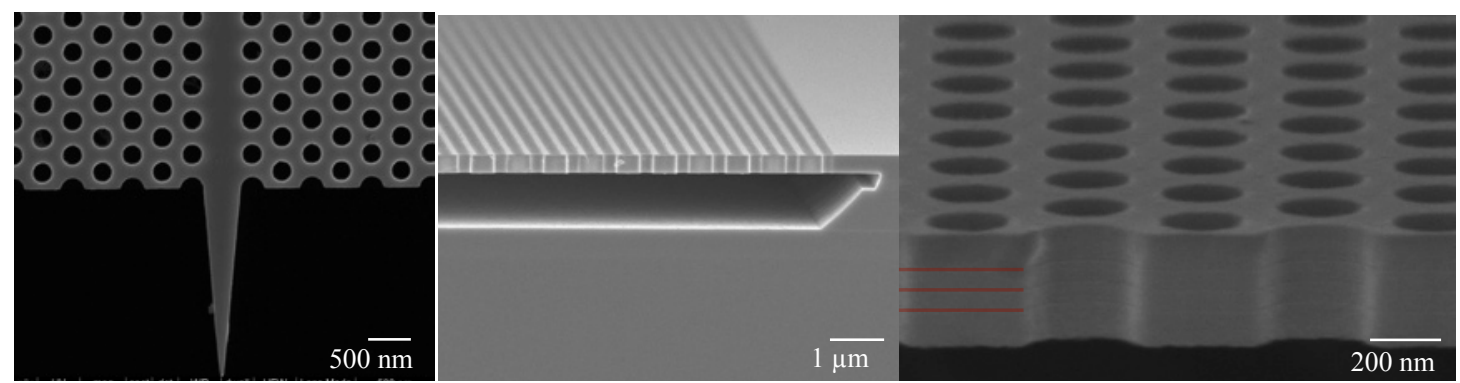

Fig. 1. SEM images of the fabricated devices. a) top view of the photonic crystal waveguide with taper b) a cross section view of the slab. There is a $1 \mu \mathrm{m}$ thick air-gap under the $330 \mathrm{~nm}$ thick active membrane. c) Zoom-in showing three layers of QD incorporated in the slab. The red lines are there for guiding the eye. 
The patterning is done using an e-beam writer (JEOL-JBX9300FS) on positive resist (ZEP520A). The pattern is transferred to $200 \mathrm{~nm} \mathrm{Si}_{3} \mathrm{~N}_{4}$ by $\mathrm{CHF}_{3} / \mathrm{O}_{2} \mathrm{RIE}$ and further transferred to the semiconductor by cyclic $\mathrm{CH}_{4} / \mathrm{H}_{2}-\mathrm{O}_{2}$ RIE. A membrane structure was finally formed with $\mathrm{HCl}$ selective wet-etching of the underlying material. Typical thickness t, lattice constant a, and airhole diameter $2 \mathrm{r}$ are $0.330,0.380$, and $0.200 \mu \mathrm{m}$ respectively. The spectral position of the slow light regime was controlled by changing the lattice constant, keeping the relation $r=0.26$ a between samples. Scanning electron microscope (SEM) images of the fabricated samples can be seen in Figure 1. Both in- and output facets of the $1 \mathrm{~mm}$ long waveguides were equipped with inverted tapers to improve coupling and reduce reflection [11]. By using tapers the Fabry-Pérot oscillations which are caused by reflection at the end facets are suppressed, shown in Figure $2 \mathrm{a}$ ). The total transmission through our $1 \mathrm{~mm}$ long 1QW device with tapers is $5.6 \%$.
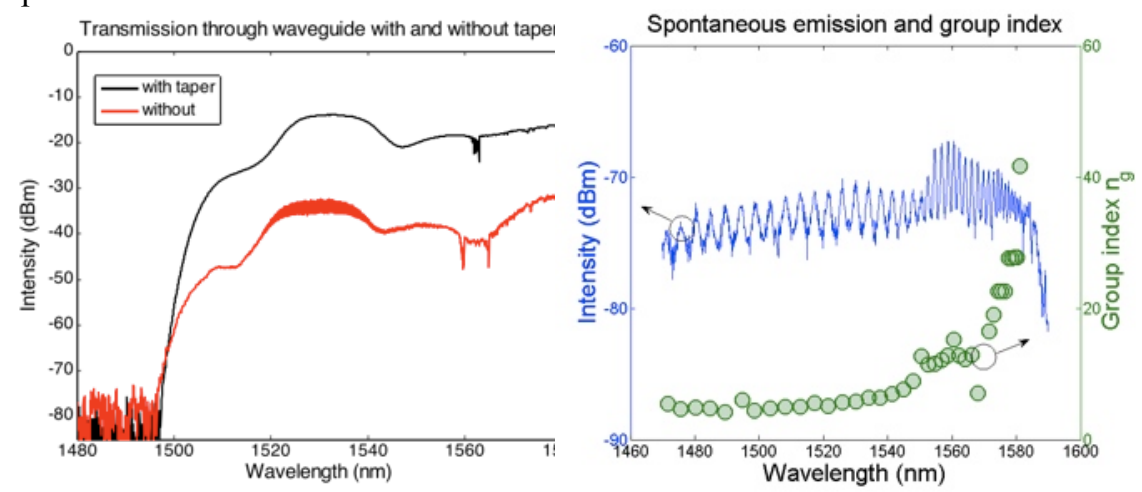

Fig. 2. a) Transmission with and without taper showing the improved transmission and suppression of fringes. b) Spontaneous emission from a $50 \mu \mathrm{m}$ short $\mathrm{PhC}$ waveguide with flat end facets $(\mathrm{RBW}=0.5 \mathrm{~nm})$ and the derived group index.

We measured the group velocity using a short $(50 \mu \mathrm{m}) 1 \mathrm{QW}$ PhC waveguide of the same design but with flat cleaves. The flat end facets promote Fabry-Pérot oscillations from which the group velocity may be derived, and the shorter PhC ensures better resolved peaks on the optical spectrum analyzer. The amplified spontaneous emission from the short 1QW PhC waveguide and the calculated group index is shown in Figure $2 \mathrm{~b}$ ). Using the wavelength spacing $\Delta \lambda$ between adjacent Fabry-Pérot peaks and the length $\mathrm{L}$, the group index was calculated using $\mathrm{n}_{\mathrm{g}}=\lambda^{2} /(2 \mathrm{~L} \Delta \lambda)$. The decrease in the group velocity is significant close to the band edge, where the groupvelocities on the order of $\mathrm{v}_{\mathrm{g}}=\mathrm{c} / \mathrm{n}_{\mathrm{g}}=\mathrm{c} / 40$ were achieved, which is a reasonable range to limit the influence of disorder effects [2].

\section{Gain measurement - method and result}

In all the gain measurements, the light from a tunable $\mathrm{cw}$ laser source was polarization controlled and transmitted through the device using lensed single mode fiber. The pump wavelength was $980 \mathrm{~nm}$ and the spot size of the pump light was $1000 \mu \mathrm{m} \times 5-7 \mu \mathrm{m}$, covering the entire length of the waveguide. Due to poor thermal conductivity of the quaternary material in combination with the free standing membrane quite severe pump-induced heating effects can be experienced. The measurements were performed at room temperature and in order to reduce heating effects the pump was pulsed. The pulse width and the repetition cycle were $0.5 \mu \mathrm{s}$ and $25 \mu \mathrm{s}$, respectively, and the maximum injected peak power was $200 \mathrm{~mW}$.

After filtering out the scattered pump light from the transmitted light, it is detected with an InGaAs detector using lock-in detection. In this scheme the change of transmission induced by the optical pump is measured, hence the absolute gain could not be measured. Instead, a relative gain was derived. The transmission at different pump powers was normalized with the transmission at the lowest measurable pump power: Relative Gain=10 $\log _{10}(($ transpump-ASE)/translowest pump), ASE being the amplified spontaneous emission. This way, the increased transmission with pump-power could be studied at different wavelengths of the propagating light, see Figure 3. Assuming the material gain to be constant over the wavelength region under test, the gain enhancement seen in Figure 3 is a contribution from the $\mathrm{PC}$ dispersion. There is a spectral offset between the enhanced gain and the material gain peak in all the samples.

Gain measurements were performed on three photonic crystal waveguides with different gain material and the results are consistent; all show enhanced gain for wavelengths in the slow-light regime of the PC. In the different samples maximum gain was measured at the wavelengths 1600,1580 and $1598 \mathrm{~nm}$ respectively, which all coincide with the calculated slow-down region for each corresponding photonic crystal design. The gain was expected to be greater for a multi QW sample than for a single QW, however, due to increased absorption of the pump this might require higher pump power. 


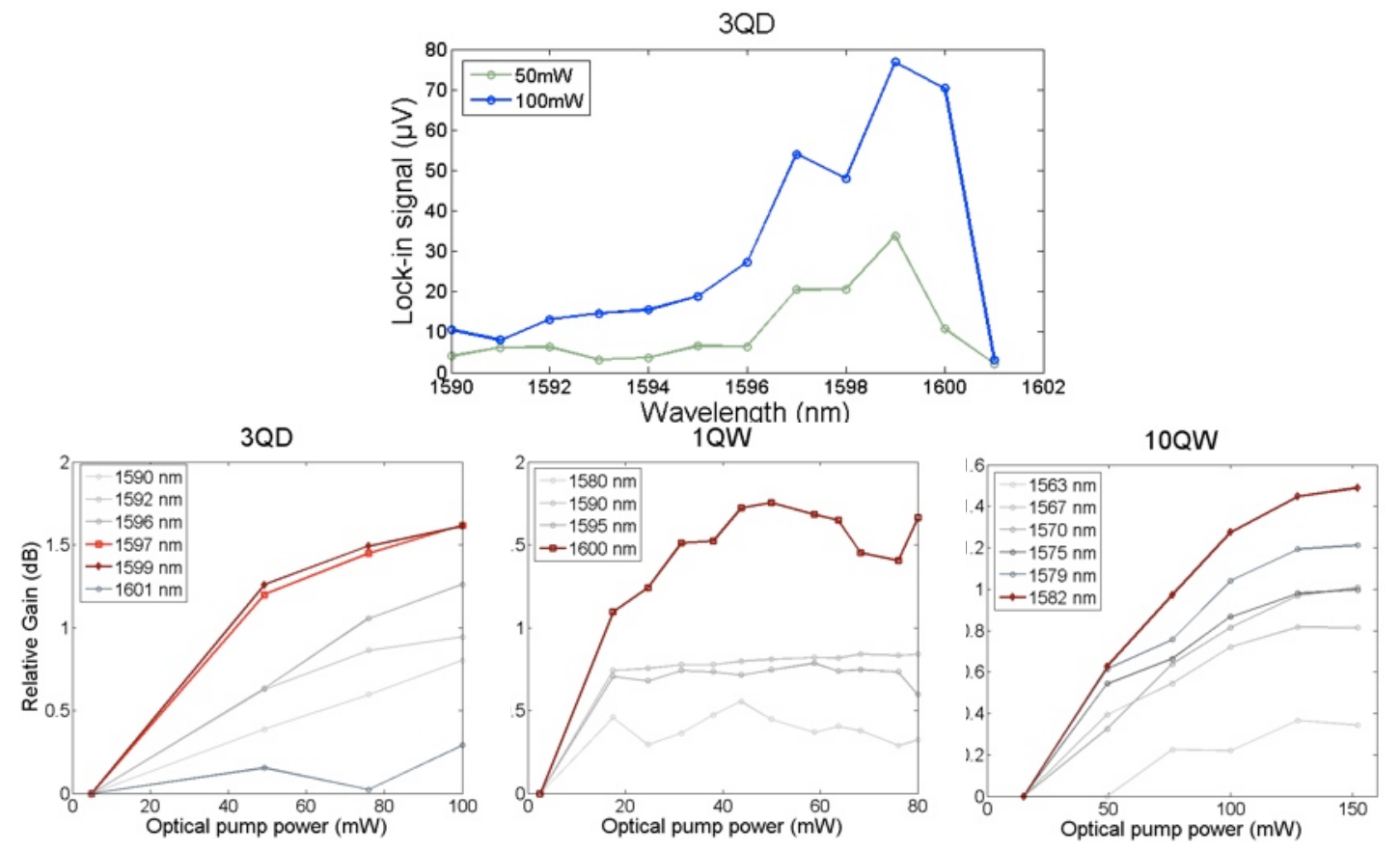

Fig. 3. a) Change of transmission for the 3QD PC waveguide at two different pump powers. b) Relative gain measurements of $3 \mathrm{QD}, 1 \mathrm{QW}$ and $10 \mathrm{QW}$ PC waveguides respectively. All showing enhanced gain in the slow light region of the PC.

\section{Conclusions}

We fabricated slow light photonic crystal slab waveguides, incorporating a single QW layer, multiple layers of QWs or multiple layers of QDs. All waveguides have taper structures at input and output to minimize insertion loss and suppress residual reflection. We show that the gain is enhanced in the slow light regime. The ability to freely tailor the dispersion in a semiconductor optical amplifier makes it possible to raise the optical gain considerably over a certain bandwidth. These results are promising for short and efficient semiconductor optical amplifiers. This effect will also benefit other devices, such as mode locked lasers, utilizing QD and QW for the gain and absorber regions.

\section{References}

[1] T. F. Krauss, "Slow light in photonic crystal waveguides," Journal of Physics D: Applied Physics 40, 2666-2670 (2007).

[2]J. Li, T.P. White, L. O'faolain, A. Gomez-iglesias, and T.F. Krauss, "Systematic design of flat band slow light in photonic crystal waveguides," Opt. Express 16, 6227-6232 (2008).

[3] E. Mizuta, H. Watanabe and T. Baba, "All Semiconductor Low- $\Delta$ Photonic Crystal Waveguide for Semiconductor Optical Amplifier," Japanese Journal of Applied Physics 45, 6116-6120 (2006).

[4] O. Painter, R. K. Lee, A. Scherer, A. Yariv, J. D. O'Brien, P. D. Dapkus, I. Kim, "Two-Dimensional Photonic Band-Gap Defect Mode Laser," Science 11, 1819-1821 (1999).

[5]K. Inoshita and T. Baba, "Room-Temperature Lasing Characteristics of Bend and Branch in Photonic Crystal Waveguide," Japanese Journal of Applied Physics 42, 6887-6891 (2003).

[6]H. Watanabe and T. Baba, "High-efficiency photonic crystal microlaser integrated with a passive waveguide," Optics express 16, 2694-8 (2008).

[7]A. Sugitatsu and S. Noda, "Room temperature operation of 2D photonic crystal slab defect-waveguide laser with optical pump," Electronics Letters 39, 213-215 (2003).

[8]K. Inoue, H. Sasaki, K. Ishida, Y. Sugimoto, N. Ikeda, Y. Tanaka, S. Ohkouchi, Y. Nakamura, and K. Asakawa, "InAs quantum-dot laser utilizing GaAs photonic-crystal line-defect waveguide.," Optics express 12, 5502-5509 (2004).

[9]K. Kiyota, T. Kise, N. Yokouchi, T. Ide, and T. Baba, "Various low group velocity effects in photonic crystal line defect waveguides and their demonstration by laser oscillation," Applied Physics Letters 88, 201904 (2006).

[10]X. Ming-Xin, Z. Wan-Hua, Z. Wen-Jun, C. Wei, L. An-Jin, and W. Hai-Ling, "Slow Light Effect and Multimode Lasing in a Photonic Crystal Waveguide Microlaser," Chinese Physics Letters 27, 024213 (2010).

[11] Q. V. Tran, S. Combrié, P. Colman, and A. De Rossi, "Photonic crystal membrane waveguides with low insertion losses," Applied Physics Letters 95, 061105 (2009). 